\title{
Defining pregnancy phases with cytokine shift
}

\author{
Sulagna Dutta* and Pallav Sengupta \\ Physiology, Lincoln University College, Malaysia
}

\begin{abstract}
Pregnancy represents an integrated orchestration of physiological processes in order to establish systemic, neuroendocrine and immunological harmony facilitating the semi-allogenic fetal growth in the maternal entity. According to the cytokine profile alterations in immune mediation of pregnancy, the later can be demarcated into three phases. The first immune phase of pregnancy, being dominated by pro-inflammatory cytokines, suffers from inflammatory propagations to enable successful implantation. In the second phase, a probable shift from pro-inflammatory Th1 cytokines to anti-inflammatory Th2 cytokines establishes symbiotic existence of fetal with maternal components ensuring robust fetal development. The final immune phase of pregnancy is again characterized by induction of inflammatory and cytolytic cytokines reinforcing inflammatory scenario to facilitate parturition. The present article, thus, compartmentalizes and simplifies the concept of immune regulation of pregnancy based on the panoply of activated cytokines.
\end{abstract}

\section{Introduction}

Pregnancy is complicated yet neatly regulated state where pro inflammatory mediators and anti-inflammatory mediators play their delicate role to establish, sustain and successfully complete the physiological processes owing to pregnancy. While inflammation is essential for implantation mechanism, immune tolerance is required to keep the semi-allogenic fetus robust and growing. Pregnancy may be alienated into three distinct immunological phases according to its regulation via different sets of cytokines $[1,2]$. The initial phase of pregnancy is marked by inflammatory responses required for implantation of the trophoblastic cells into endometrial cells. The second phase is a state of anti-inflammation needed for symbiotic coexistence of the mother and fetus ensuring proper growth and development of the later.

Immune cells, activated during different phases of pregnancy such as, macrophages, T cells, B cells, NK cells etc., function in an orchestral manner. Pregnancy is thought to possess a polarized form of immune response mainly orchestrated by the T cells [3]. T helper cells (CD4+) may be subdivided into T helper 1 cells (Th1) and T helper 2 cells (Th2) based on cytokine production. Th1 cells when activated trigger inflammation by secreting pro inflammatory cytokines such as tumor necrosis factor alpha (TNF- $\alpha$ ) and interferon gamma (IFN- $\gamma$ ), whereas the Th2 cells aid wound healing and immune tolerance via antiinflammatory cytokines such as interleukin 10 (IL-10), IL-4, and IL-13 [4]. Wegmann and colleagues had put forth the concept of a cytokine shift during pregnancy from a T helper 1 (Th1) cascade to a T helper 2 (Th2) ones to induce maternal tolerance and immune suppression [5]. But in order for the fetal blastocyst to implant itself in the maternal uterine walls, the embryo is compelled to invade through the uterine lining thereby damaging the underlying maternal tissue, and disrupting the blood vessels to obtain an adequate blood supply for itself. These invading physiological activities establish maternal inflammatory response to secure repair mechanism of the uterus and also for the sake of removal of dying maternal cells [2]. Hence, this article attempts to concisely present the three immune regulatory phases of pregnancy based on the role of pro and anti-inflammatory cytokines.

\section{Phase 1: Implantation representing an 'open wound'}

During post coitus period, seminal plasma plays a vital immunoregulatory function in the establishment of pregnancy triggering inflammatory reaction in endometrium. This leads in the recruitment of a panoply of immune effector cells to the site of implantation. All the mediators of these inflammatory response remain elusive, but actions of cytokines in concert with prostaglandins and various steroid-binding proteins have been established [6]. TGF-b1 in seminal plasma is reportedly one of the prime agents regulating this inflammatory condition. Human semen is a repertoire of cytokines, including IL-2, IL-4, IL-1a/b, IL-6, IL-10, IL-8, IL-11, IL-12, TNFa, IFN $\gamma$, monocyte chemotactic and activating factor, MIF, vascular endothelial growth factor (VEGF)regulated on activation, normal T cell expressed and secreted (RANTES), macrophage colony-stimulating factor (CSF1), granulocyte colony stimulating factor (G-CSF), macrophage-CSF, stem cell factor, etc. [6]. Eotaxin, macrophage inflammatory proteins, monocyte chemotactic protein (MCP)-1, keratinocyte-derived chemokine, and IL-9 may also have immunoregulatory roles in postcoital uterus [7]. These inflammatory responses, besides facilitating sperm survival and later their removal from the female reproductive system [8], also regulate uterine immune functions, such as granulocyte trafficking, recruitment, activation and presentation of paternal antigens, thereby mediating immunosurveillance in favor of embryo implantation and the establishment of pregnancy [6].

Implantation, placentation as well as the first and early second trimester of pregnancy bear a resemblance to the inflammatory response to 'an open wound'. During the first stage, the blastocyst invades into the uterine epithelial lining to implant itself, damaging

Correspondence to: Sulagna Dutta, Ph.D., Lecturer, Physiology Unit, Faculty of Medicine, Lincoln University College, Malaysia, Tel: 0111-420 8705; E-mail: sulagna_dutta11@yahoo.com

Key words: cytokines, inflammation, pregnancy

Received: October 25, 2017; Accepted: November 13, 2017; Published: November 17, 2017 
the endometrial tissue and the trophoblast of the blastocyst replace the uterine endothelium and maternal blood vessels (vascular smooth muscle) to adapt adequate blood supply [9]. These processes result in infiltration of various invading cells, dying cells as well as repairing cells. Thus an inflammatory response is needed to repair the damaged uterine epithelium and for removing cellular debris. During this time, mother's body is struggling to become immunologically accustomed to the presence of the fetus (in addition to other physiological changes such as alterations in endocrine regulation) [10-12]. Pre-implanted embryos produce various cytokines (IL-6, EGF, basic fibroblast growth factor (bFGF), which aid embryo-maternal amalgamation at the implantation sites. Blastocyst attachment to the uterine wall reportedly involves steroid-hormone sensitive secretion of heparin binding EGF via the uterine epithelium which also bring about blastocyst outgrowth. Implantation is also mediated through the actions of critical cytokines such as leukemia inhibitory factor (LIF) like cytokines and IL-1 system components [13]. The decidualization process involves cytokines (IL11, LIF, activin A and monoclonal non-specific suppressor factor-b) for tissue differentiation [14]. Endometrial cytokine production is strictly regulated to recruit maternal uterine natural killer (NK) cells and macrophages (through chemotaxis and 1-selectin expression) to aid inflammation and to facilitate invasion of fetal trophoblast through the decidua and vasculature. They differentiate to provide an endothelium like surface to maternal blood while remodeling maternal spiral arteries to supply nutrition and oxygen to the fetus [15]. Vascularization owing to placental development is a cytokine-regulated process too, including placental growth factor (PlGF), vascular endothelial growth factor (VEGF) and the IL-12/IL-15/IL-18 system acting as tissue remodeling, angiogenic and NK cell regulatory factors [16,17]. Strict regulation of the actions of uterine NK is imperative, since IFN- $\gamma$, secreted by them, helps in trophoblast invasion via apoptosis induction. Activated leukocytes recruited and activated in the decidua further contribute to the established inflammatory microenvironment generating array of regulatory cytokines [14]. Thus both the processes of implantation and placentation are proinflammatory processes involving various growth factors and pro inflammatory cytokines but there is also a coexisting regulatory arm to this inflammatory response in the maternal decidua, where some leukocytes induce immunotolerant natural killer (NK) cells, CD14 p monocytes and other cells producing anti-inflammatory mediators such as G-CSF, IL-6 [18].

\section{Phase 2: Adaptation to semi allogenic fetus}

The second immunological phase is considered a soothing pregnancy period for the mother. This is a period when the fetus rapidly grows establishing a symbiosis with the maternal components. Thus, the mother, placenta and fetus are adapted to each other, and the predominant immunological process strive to induce an antiinflammatory state. The conventional concept of this anti-inflammatory state is cytokine shift from inflammatory Th1 cytokines to antiinflammatory Th2 cytokine via regulation of NK and lymphokineactivated cells [19]. Modulatory cytokines (IL-3, IL-4, IL-5, IL-10) are observed at the foetomaternal interface which minimize Th1type responses [20] and the preponderant contribution of Th2-type cytokines during this second phase of pregnancy, largely owe to nonlymphoid trophoblast and placental/ decidual tissues [21]. In addition to establish immune tolerance towards the fetal allograft, cytokines in this phase also promote uterine expansion. The mechanical stretch in the uterine wall by the growing gestational sac may lead to cytokine production transforming growth factor (TGF- $\beta 3$ ) which in turn may accelerate uterine growth $[22,23]$. Endometrium, at this expanding phase, also induce IL-1a, IL-1b, IL- 6 and IL- 8 for the sustenance of anti-inflammatory state and development of the uterine and fetal tissue [23]. Many other cytokines (e.g. TGF- $\beta$ ) are implicated in embryo/ fetal developmental through regulatory processes such as epithelialmesenchymal transformation. They also manage erythropoiesis, cardiovascular/skeletal development, craniofacial morphogenesis and axial patterning [24-26].

\section{Phase 3: Renewal of inflammation for parturition}

The last immunological phase of pregnancy marks the completion of the fetal development. Now the mother requires to deliver the baby by the process of parturition which is mediated through renewed inflammation. Thus the anti-inflammatory immune state is reversed following the onset of labor [19]. The precise immune mechanisms of regulation of labor are not completely transparent, but occurrence of an inflammatory sequence by coordinated production of cytokines and prostaglandins has been accepted [27]. Firstly, IL-6 and GM-CSF in the late pregnancy may help in the final stages of lung maturation of the fetus [23]. Decline in maternal progesterone and pulmonary maturation lead to elevation of surfactant protein-A (SP-A) and phospholipid release (e.g. arachidonic acid to synthesize prostaglandin). SP-A activates fetal amniotic macrophages which migrate to uterine wall and generate IL$1 \mathrm{~b}$ thereby triggering an inflammatory/prostaglandin cascade resulting in uterine contractility [28]. Myometrial leukocytic incursion and relocation also contributes to IL-1b, IL- 6 and IL-8 production which are inflammatory and chemotactic [23]. These roles of cytokines in promoting uterine smooth muscle contraction is evident as they are reported to increase intracellular calcium, phospholipase activity, and induce prostaglandin and oxytocin receptor generation in the human myometrial cells in vitro [23], which are essential to bring about uterine contraction during parturition. Moreover, cytokines (IL-1 and TNF- $\alpha$ ) aid arachidonic acid release to induce further prostaglandin biosynthesis [29]. This inflammatory process set at this phase, also affect the chorioamniotic membranes [30]. The gestational membrane cytokine production elevates matrix metalloproteinase (MMP) expression to mediate extracellular matrix remodeling and weakening by triggering collagen breakdown leading to rupture and dissociation of the fetal membranes [31]. While the epithelium of the cervix also emanate an array of cytokines (M-CSF (microphage-colony stimulating factor), TGF- $\beta 1$, IL- $1 \mathrm{a} / \mathrm{b}, \mathrm{IL}-6, \mathrm{IL}-7, \mathrm{IL}-8$, , RANTES) and is still responsive to exogenous IFN- $\gamma$ and TNF- $\alpha$ [23]. During this phase, remodeling of cervix via inflammatory progression is mediated by the fibroblasts by upregulation of cytokines (IL-6, IL- 8), matrix metalloprotease-1 (MMP-1) and MMP-3 and inhibition of their tissue inhibitors (tissue inhibitors of metalloprotease, TIMPs) [31]. Thus, parturition is governed by an infiltration of active immune cells into the myometrium to stimulate an inflammatory process which promotes uterine contraction, expulsion of the baby and rejection of the placenta $[10,27]$.

\section{Conclusion}

Pregnancy possesses both pro-inflammatory and antiinflammatory immune responses depending upon the stages of gestation. Cytokines are the major immune regulators in pregnancy, mediating gametogenesis, uterine receptivity, implantation reactions, embryogenesis and fetal development and the onset of parturition either by triggering inflammatory processes or by inducing immune tolerance. Based on the immune regulation and types of regulating cytokines, pregnancy can be demarcated into three distinct phases. In particular, cytokines govern the immunological balance required 
for evoking inflammation at the first phase during invasion of semiallogenic fetal cells into endometrial cells, establishment of symbiosis between fetus and mother at the second phase, by promoting fetal allograft tolerance and again inducing inflammatory processes at the final phase in order to detach fetal tissues from that of the mother's. This article thus provides a simplified concept to aid greater understanding of the complex cytokines network orchestrated to mediate physiological processes in every phase of pregnancy.

\section{References}

1. Mor G, Koga K (2008) Macrophages and pregnancy. Reprod Sci 15: 435-436. [Crossref]

2. Mor G (2007) Pregnancy reconcieved. Natural History. 116:36-41.

3. Reid TM (1998) Striking a balance in maternal immune response to infection. Lancet. 6:1670.

4. Mosmann TR, Sad S (1996) The expanding universe of T-cell subsets: Th1, Th2 and more. Immunol Today 17: 138-146. [Crossref]

5. Wegmann TG, Lin H, Guilbert L, Mosmann TR (1993) Bidirectional cytokine interactions in the maternal-fetal relationship: is successful pregnancy a TH2 phenomenon? Immunol Today. 14:353-6.

6. Gopichandran N1, Ekbote UV, Walker JJ, Brooke D, Orsi NM (2006) Multiplex determination of murine seminal fluid cytokine profiles. Reproduction 131: 613-621. [Crossref]

7. Robertson SA1, Mau VJ, Hudson SN, Tremellen KP (1997) Cytokine-leukocyte networks and the establishment of pregnancy. Am J Reprod Immunol 37: 438-442. [Crossref]

8. Denison FC, Grant VE, Calder AA, Kelly RW (1999) Seminal plasma components stimulate interleukin-8 and interleukin-10 release. Mol Hum Reprod. 5:220-6.

9. Dekel N, Gnainsky Y, Granot I, Mor G (2010) Inflammation and implantation. Am J Reprod Immunol. 63:17-21.

10. Mor G, Abrahams V: Immunology of implantation. In Immunology and Allergy Clinics. Edited by Arici A. Philadelphia: W.B. Saunders Company; 2002: 545-65

11. Dutta S, Sengupta P (2017) Yoga escalates female reproductive health during pregnancy. J Pregnancy Reprod. 1:1.

12. Sengupta $P$ (2014) The bliss yoga inculcates during the different stages of pregnancy. International Journal of Pharmacy and Pharmaceutical Sciences 6:86-87.

13. Sharkey A (1998) Cytokines and implantation. Rev Reprod 3: 52-61. [Crossref]

14. Salamonsen LA, Hannan NJ, Dimitriadis E (2007) Cytokines and chemokines during human embryo implantation: roles in implantation and early placentation. Semin Reprod Med. 25:437-44. [Crossref]

15. Zhou Y, Genbacev O, Fisher SJ (2003) The human placenta remodels the uterus by using a combination of molecules that govern vasculogenesis or leukocyte extravasation. Ann N Y Acad Sci 995: 73-83. [Crossref]
16. Torry DS, Hinrichs M, Torry RJ (2004) Determinants of placental vascularity. Am J Reprod Immunol 51: 257-268. [Crossref]

17. Králícková M, Síma P, Rokyta Z (2005) Role of the leukemia-inhibitory factor gene mutations in infertile women: the embryo-endometrial cytokine cross talk during implantation--a delicate homeostatic equilibrium. Folia Microbiol (Praha) 50: 179186. [Crossref]

18. Engert S, Rieger L, Kapp M, Becker JC, Dietl J (2007) Profiling chemokines, cytokines and growth factors in human early pregnancy decidua by protein array. Am J Reprod Immunol. 58:129-37. [Crossref]

19. Raghupathy R (1997) Th1-type immunity is incompatible with successful pregnancy. Immunol Today. 18:478-82. [Crossref]

20. Carp H1 (2004) Cytokines in recurrent miscarriage. Lupus 13: 630-634. [Crossref]

21. Chaouat G, Cayol V, Mairovitz V, Dubanchet S (1999) Localization of the Th2 cytokines IL-3, IL-4, IL-10 at the fetomaternal interface during human and murine pregnancy and lack of requirement for Fas/Fas ligand interaction for a successful allogeneic pregnancy. Am J Reprod Immunol. 42:1-13. [Crossref]

22. Shynlova O, Tsui P, Dorogin A, Langille BL, Lye SJ (2007) The expression of transforming growth factor beta in pregnant rat myometrium is hormone and stretch dependent. Reproduction 134: 503-511. [Crossref]

23. Orsi NM, Tribe RM (2008) Cytokine networks and the regulation of uterine function in pregnancy and parturition. J Neuroendocrinol 20: 462-469. [Crossref]

24. Barnett JV, Desgrosellier JS (2003) Early events in valvulogenesis: a signaling perspective. Birth Defects Res C Embryo Today. 69:58-72. [Crossref]

25. Serra R, Chang C (2003) TGF-beta signaling in human skeletal and patterning disorders. Birth Defects Res C Embryo Today. 69:333-51. [Crossref]

26. Nawshad A, LaGamba D, Hay ED (2004) Transforming growth factor beta (TGFbeta) signalling in palatal growth, apoptosis and epithelial mesenchymal transformation (EMT). Arch Oral Biol. 49:675-89. [Crossref]

27. Romero R, Espinoza J, Kusanovic JP, Gotsch F, Hassan S (2006) The preterm parturition syndrome. BJOG. 113 Suppl 3:17-42.

28. Condon JC, Jeyasuria P, Faust JM, Mendelson CR (2004) Surfactant protein secreted by the maturing mouse fetal lung acts as a hormone that signals the initiation of parturition. Proc Natl Acad Sci U S A. 101:4978-98. [Crossref]

29. Molnar M, Romero R, Hertelendy F (1993) Interleukin-1 and tumor necrosis facto stimulate arachidonic acid release and phospholipid metabolism in human myometrial cells. Am J Obstet Gynecol. 169:825-9. [Crossref]

30. Haddad R, Tromp G, Kuivaniemi H, Chaiworapongsa T, Kim YM, et al. (2006) Human spontaneous labor without histologic chorioamnionitis is characterized by an acute inflammation gene expression signature. Am J Obstet Gynecol. 195:394 e1-24. [Crossref]

31. Cockle JV, Gopichandran N, Walker JJ, Levene MI, Orsi NM (2007) Matrix metalloproteinases and their tissue inhibitors in preterm perinatal complications. Reprod Sci. 14:629-45. [Crossref]

Copyright: (C2017 Dutta S. This is an open-access article distributed under the terms of the Creative Commons Attribution License, which permits unrestricted use, distribution, and reproduction in any medium, provided the original author and source are credited. 